\title{
Percolation properties of the Wolff clusters in planar triangular spin models
}

\author{
P. W. Leung* and Christopher L. Henley \\ Laboratory of Atomic and Solid State Physics, Cornell University, Ithaca, New York 14853
}

(Received 22 August 1990)

\begin{abstract}
We formulate the Wolff algorithm as a site-bond percolation problem, apply it to the ferromagnetic and antiferromagnetic planar triangular spin models, and study the percolation critical behavior using finite-size scaling. In the former case the Wolff algorithm is successful as an accelerating algorithm, whereas in the latter case it is not. We found the percolation temperatures and the cluster exponents for both models. In the antiferromagnetic model, the percolation temperature is higher than the critical temperature of the spin system. The cluster exponents are found to be the same as the random two-dimensional (2D) percolation. In the ferromagnetic model, the percolation temperature agrees with the critical temperature, and the cluster exponents are different from the random 2D percolation, meaning that they are in different universal classes. For the ferromagnetic model we discuss the mechanism of the cluster growth in the regime of the Kosterlitz-Thouless transition. We also note a relation between the dynamic exponent and the percolation exponents.
\end{abstract}

\section{INTRODUCTION}

Ever since Swendsen and Wang ${ }^{1}$ (SW) put forward their novel algorithm for the Potts model, various algorithms have been proposed ${ }^{2-5}$ to reduce critical slowing down of spin systems using ideas from percolation theory. The original SW algorithm ${ }^{1}$ is based on the Fortuin-Kasteleyn mapping ${ }^{6}$ for site-bond percolation and the Potts model. Clusters of spins in the same states are grown and the whole clusters are flipped. Thus, a number of spins are updated in a single move and the correlation time is reduced. This was successfully applied to the ferromagnetic Potts model and the antiferromagnetic Ising model on a square lattice. ${ }^{1}$ Later Wolff ${ }^{4}$ introduced a single-cluster algorithm that, when applied to the Ising model, is similar to the SW algorithm except that only one of the SW clusters is flipped. This particular cluster is chosen with a probability proportional to its size. Wolff also introduced a generalization of the spinflip operation in the Ising model to models with continuous degree of freedom and successfully applied it to the ferromagnetic $X Y$ and Heisenberg model. ${ }^{4}$ Using a combination of the SW algorithm and the Wolff flips, Wang, Swendsen, and Kotecký ${ }^{5}$ were able to deal with the antiferromagnetic three-state Potts model on a square and simple-cubic lattice. In all of the above cases, the systems are not frustrated and the new algorithms give a significantly smaller dynamical exponent than the Metropolis algorithm. A comparison of the efficiency between the SW and Wolff algorithm for the Ising model was done by Tamayo, Brower, and Klein ${ }^{7}$ who found that, for $d>2$, the Wolff algorithm is more efficient than the $\mathrm{SW}$ algorithm.

We have been motivated by an interest in techniques for acceleration of vector spin systems with competing interactions (frustration). An alternative method, Fourier acceleration, can speed up the relaxation of the longwavelength modes of any continuous degree of freedom. It is successful in reducing critical slowing down in fer- romagnetic and other unfrustrated spin systems. ${ }^{8,9}$ However, frustrated systems commonly break discrete degrees of freedom in addition to the global continuous symmetry; in particular, the global symmetry group $\mathrm{O}(n)$ for $n$ vector spin systems contains a reflection operation which is discrete. When Fourier acceleration was applied to such a system, it was found to be ineffective in accelerating the discrete degree of freedom. ${ }^{10}$ The Wolff update is exactly a reflection, so there is an apparent possibility that it could be useful in updating these degrees of freedom. Unfortunately, our study has shown that the Wolff algorithm does not succeed in finding the right groupings of spins to be reflected.

While the previous works are concentrated on showing the efficiency of the new algorithms by finding the dynamical critical exponent, in this paper we present a study of the percolation properties of the clusters ${ }^{11}$ in the Wolff algorithm. Instead of being used as an acceleration algorithm to reduce critical slowing down, we formulate the Wolff algorithm as a site-bond percolation problem. It is applied to the planar triangular spin model with ferromagnetic (FPT) and antiferromagnetic (AFPT) interactions, the latter being a frustrated system. We will show that, for the FPT model, $T_{p}$ (the percolation temperature of the Wolff clusters) is equal to $T_{c}$ (the critical temperature of the spin system), whereas $T_{p}>T_{c}$ for the AFPT model. Thus, in one case (FPT), the Wolff algorithm is successful in reducing critical slowing down while in the other case (AFPT) it is not. We will investigate the properties of the Wolff clusters in these two cases.

\section{WOLFF ALGORITHM AS A PERCOLATION PROBLEM}

Consider a classical planar spin system with Hamiltonian

$$
H=-\beta J \sum_{\langle x y\rangle} \sigma_{x} \cdot \sigma_{y}
$$


Wolff $^{4}$ generalized the spin-flip operation in the Ising model to a continuous model by defining it to be a reflection about a direction orthogonal to a projection vector $\mathbf{r}$ in the spin space

$$
\mathcal{R}(\mathbf{r}) \sigma_{x}=\sigma_{x}-2\left(\sigma_{x} \cdot \mathbf{r}\right) \mathbf{r} .
$$

For a particular projection vector $\mathbf{r}$ which is chosen at random, we can identify the clusters in the system by visiting each nearest-neighboring bond $\langle x y\rangle$ and activating it with the probability

$P\left(\sigma_{x}, \sigma_{y}\right)=1-\exp \left\{\min \left[0,-2 \beta J\left(\mathbf{r} \cdot \boldsymbol{\sigma}_{x}\right)\left(\mathbf{r} \cdot \boldsymbol{\sigma}_{y}\right)\right]\right\}$.

We then pick a spin at random. The cluster to which it belongs is called the Wolff cluster. The Wolff algorithm of sampling the spin configurations consists of continuously generating the Wolff clusters using random projection vectors and flipping them according to Eq. (2) as soon as they are generated. In this way we generate a series of spin configurations and a distribution of Wolff clusters $n_{s}^{*}$. It can be shown ${ }^{4}$ that this satisfies the detailed balance and will sample spin configurations according to the partition function corresponding to the Hamiltonian in Eq. (1).

At high temperatures, the spins are less correlated and the cluster sizes are small. As the temperature is lowered, the spins become more ordered and the cluster sizes increase. We define the percolation temperature of the Wolff clusters $T_{p}$ as the temperature at which spanning clusters start to appear in a large system. Therefore, we have two different critical temperatures in our system: the critical temperature of the spin system $T_{c}$ and the percolation temperature of the clusters $T_{p}$. In our twodimensional (2D) models, we consider $T_{c}$ to be the Kosterlitz-Thouless $^{12}$ (KT) temperature for the FPT model. For the AFPT model, we consider $T_{c}$ to be the temperature at which both discrete and continuous degrees of freedom ${ }^{13}$ order. As in an ordinary percolation problem, we can define the following cluster properties for the Wolff clusters: the percolation probability $P_{\infty}$ which is the fraction of spins belonging to the spanning cluster, the distribution of cluster size $n_{s}=N_{s} / N$, where $N_{s}$ is the fraction of Wolff clusters with size $s$, and $N=L^{d}$ is the total number of spins in the lattice. Also, the cluster susceptibility $\chi_{p}$ is the second moment of the cluster distribution

$$
\chi_{p} \equiv \sum_{s} s^{2} n_{s} .
$$

We will write the expectations $\langle\cdots\rangle$ with respect to the Wolff process. A given Wolff cluster has one chance to be grown for each site that can be hit; thus, the probability of hitting a Wolff cluster of size $s$ is

$$
n_{s}^{*}=\frac{s}{N} N_{s}=s n_{s},
$$

i.e., the clusters are hit with probability proportional to their sizes. Then we can write

$$
\chi_{p}=\sum_{s} s n_{s}^{*}=\langle s\rangle \text {. }
$$

Here the temperature of the system plays the role of the percolation probability $p$ in the ordinary percolation problem. The system is above the percolation threshold when $T$ is below $T_{p}$ and vice versa.

To make the computational problem well defined, we need a working definition of $P_{\infty}$ when $L<\infty$. If we found all clusters simultaneously as in $\mathrm{SW},{ }^{1}$ then we could use the standard approach of taking the largest cluster as the "infinite" spanning cluster. However, with the algorithm of Ref. 4 , we only generate one cluster at a time; therefore, we have chosen to extract $P_{\infty}$ from the function $n_{s}^{*}$. We suppose there is a cutoff cluster size $s_{c}$, above which the Wolff clusters are considered to be spanning clusters. Now, in the limit $L \rightarrow \infty$, we note that $P_{\infty}$ is related to $n_{s}^{*}$ in two independent ways; we generalize these ways to finite $L$, and then we fix $s_{c}$ by requiring that both ways give the same value of $P_{\infty}$. The two ways are the following: (i) The size of the spanning cluster is $P_{\infty} N$; thus, we require

$$
P_{\infty}=\frac{1}{N}\langle s\rangle_{s>s_{c}}=\frac{1}{N} \frac{\sum_{s_{c}}^{N} s n_{s}^{*}}{\sum_{s_{c}}^{N} n_{s}^{*}} .
$$

(ii) On the other hand, since $P_{\infty}$ is the fraction of all spins which belong to the spanning cluster, the probability that a Wolff hit is on the spanning cluster is $P_{\infty}$. Thus, we require

$$
P_{\infty}=\operatorname{Prob}\left(s>s_{c}\right)=\sum_{s_{c}}^{N} n_{s}^{*} .
$$

[Note the normalization $\sum_{1}^{N} n_{s}^{*}=1$ follows from (5).]

Note that $P_{\infty}$ from Eq. (7) increases as $s_{c}$ increases, whereas $P_{\infty}$ from Eq. (8) decreases as $s_{c}$ increases, so there is a unique solution of $s_{c}$ for which (7) and (8) are equal. This uniquely defines an estimator $\widehat{P}_{\infty}$ from the distribution of Wolff clusters $n_{s}^{*}$.

As in ordinary percolation problems, we make the following scaling assumptions:

$$
\begin{aligned}
& P_{\infty}=L^{-\beta_{p} / v_{p}} f\left(t L^{1 / v_{p}}\right), \\
& \chi_{p}=L^{\gamma_{p} / v_{p}} g\left(t L^{1 / v_{p}}\right), \\
& n_{s}^{*}=s^{-\tau_{p}^{*}} h\left(t s^{\sigma_{p}}, t L^{1 / v_{p}}\right),
\end{aligned}
$$

where $t=\left|1-T / T_{p}\right|$ and

$$
\tau_{p}^{*}=\tau_{p}-1 \text {. }
$$

Equation (12) results from Eq. (5) and so the Wolff exponent $\tau_{p}^{*}$ is smaller than the cluster exponent $\tau_{p}$ by 1 . Observe that in the limit $t \rightarrow 0$, Eq. (11) implies

$$
n_{s}^{*}=s^{-\tau_{p}^{*}} \widetilde{h}\left(t s^{\sigma_{p}}\right) \text {. }
$$

This is consistent with the fractal scaling

$$
s \sim R^{D}
$$

for clusters of diameter $R$ only if the fractal dimension $D$ 
obeys the relation $D v \sigma=1$. It can be shown by inserting (11) into (7) and (8) that our estimator $\widehat{\boldsymbol{P}}_{\infty}$ indeed satisfies (9).

\section{RESULTS}

We now consider an AFPT model with Hamiltonian (1) and $J=-1$. The $T_{c}$ for this system was previously determined to be $0.505 \pm 0.005 .^{13}$ We generate the Wolff clusters according to the algorithm in Ref. 4, which is an equivalent, but more efficient, version of the algorithm described above. A "Wolff step" consists of generating a Wolff cluster and flipping it to obtain a new spin configuration; the number of spins updated in one Wolff step is equal to the size of the Wolff cluster. The following runs are for 100000 Wolff steps. The system sizes are $L=12,18,24,36,48$, and 72 , where $N=L^{2}$. The spin configurations are in a parallelogram cell with periodic boundary conditions. Figure 1 shows a typical distribution of the cluster sizes at a temperature above $T_{p}$, (i.e., below percolation threshold). For intermediate cluster sizes, $n_{s}^{*}$ follows a power law. Figure 2 shows the scaling plots for $P_{\infty}, \chi_{p}$, and $n_{s}^{*}$. It is clear that the distribution of the Wolff clusters depends on the size $L$. Hence, there are two arguments in the scaling function $h$ in Eq. (11). But, for $s<L^{d}$, one will expect that the dependence of $n_{s}^{*}$ on $L$ is not important. In Fig. 2(c), only the data points for $L=72$ (which is our largest system) are plotted. $T_{p}$ and the critical exponents are found by adjusting their values to achieve optimal collapsing of the data points into a single curve. Their uncertainties are estimated by varying their values and visually determining the maximum deviation so that the data collapsing is still acceptable. From the scaling plots we find that $T_{p}=1.037 \pm 0.002$. The cluster exponents are tabulated in Table I. Note that values of $v_{p}$ are independently determined from Figs. 2(a) and 2(b), and they turn out to be the same. Also listed in Table I are the critical exponents for the $2 \mathrm{D}$ random percolation calculated from

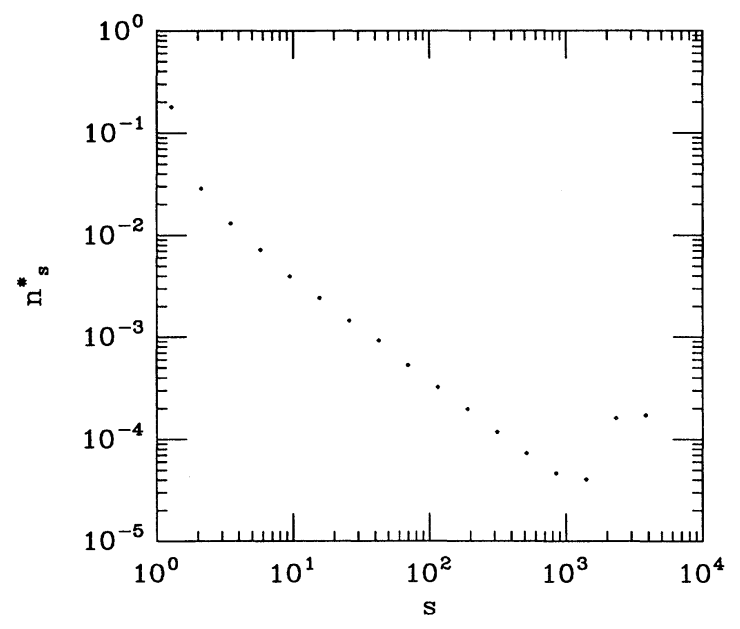

FIG. 1. Distribution of Wolff clusters $n_{s}^{*}$ for the AFPT model with $L=72$ at $T=1.04$.
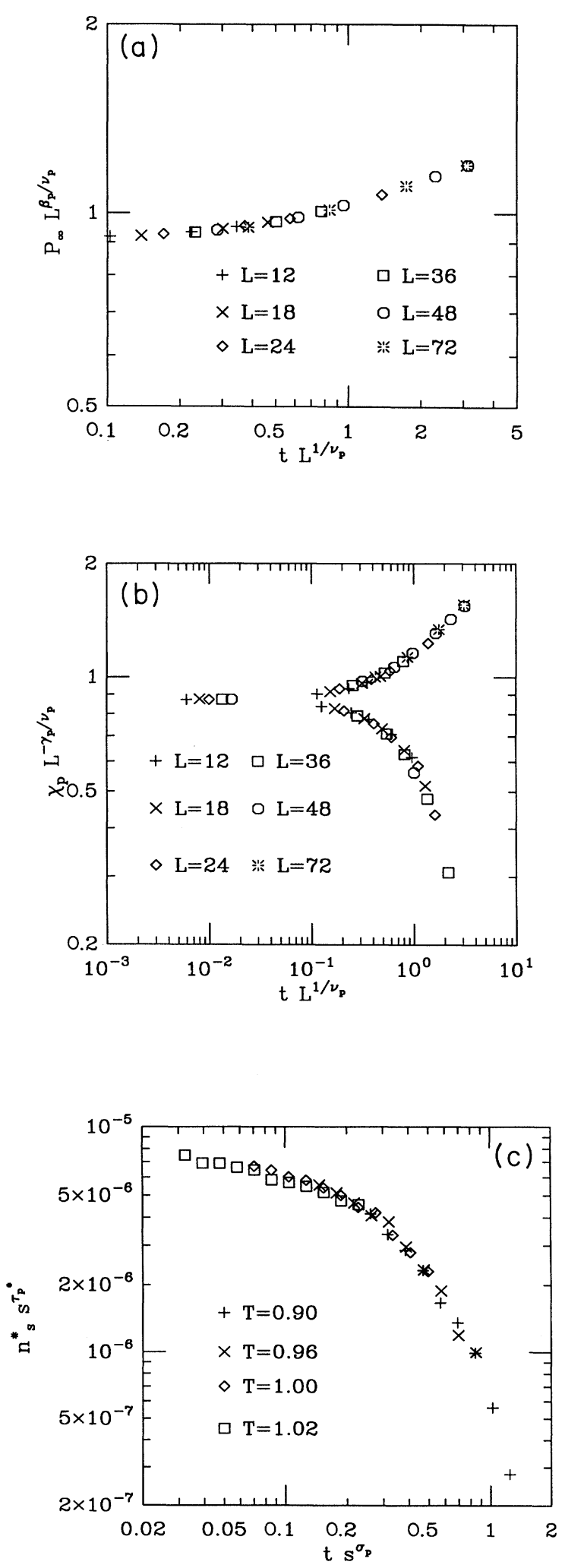

FIG. 2. Finite-size scaling plots for the AFPT model: (a) $\boldsymbol{P}_{\infty}$, (b) $\chi_{p}$; the upper and lower branches are for $T<T_{p}$ and $T>T_{p}$, respectively. (c) Scaling plot (for $L=72$ only) of cluster size distribution $n_{s}^{*}$. 
TABLE I. Critical exponents for the Wolff clusters as found from the scaling plots in Figs. 2 and 3 for the AFPT and FPT models, respectively. $D$ is the fractal dimension. The values for 2D percolation are from Ref. 17. $\tau_{p}$ is obtained from $\tau_{p}^{*}$ through Eq. (12), $D$ is calculated from Eq. (17). The other exponents are obtained from the scaling plots.

\begin{tabular}{cllc}
\hline Exponent & \multicolumn{1}{c}{ AFPT } & \multicolumn{1}{c}{ FPT } & 2D percolation \\
\hline$\beta_{p}$ & $0.140 \pm 0.004$ & $0.25 \pm 0.03$ & $\frac{5}{36}=0.139$ \\
$v_{p}$ & $1.355 \pm 0.005$ & $2.5 \pm 0.2$ & $\frac{4}{3}=1.333$ \\
$\gamma_{p}$ & $2.41 \pm 0.02$ & $4.5 \pm 0.4$ & $\frac{43}{18}=2.389$ \\
$\tau_{p}$ & $2.01 \pm 0.02$ & & $\frac{187}{91}=2.055$ \\
$\sigma_{p}$ & $0.39 \pm 0.02$ & & $\frac{36}{91}=0.396$ \\
$D$ & $1.88 \pm 0.02$ & $1.90 \pm 0.01$ & $\frac{91}{48}=1.896$ \\
\hline \hline
\end{tabular}

the conjectures of den Nijs, ${ }^{14}$ Pearson, ${ }^{15}$ and Nienhuis et $a l .{ }^{16}$ These values are believed to be exact. ${ }^{17} \mathrm{We}$ observe that they agree quite well. Thus, the exponents numerically obey the usual scaling laws

$$
\begin{aligned}
& \beta_{p}=\left(\tau_{p}-2\right) / \sigma_{p}, \\
& -\gamma_{p}=\left(\tau_{p}-3\right) / \sigma_{p},
\end{aligned}
$$
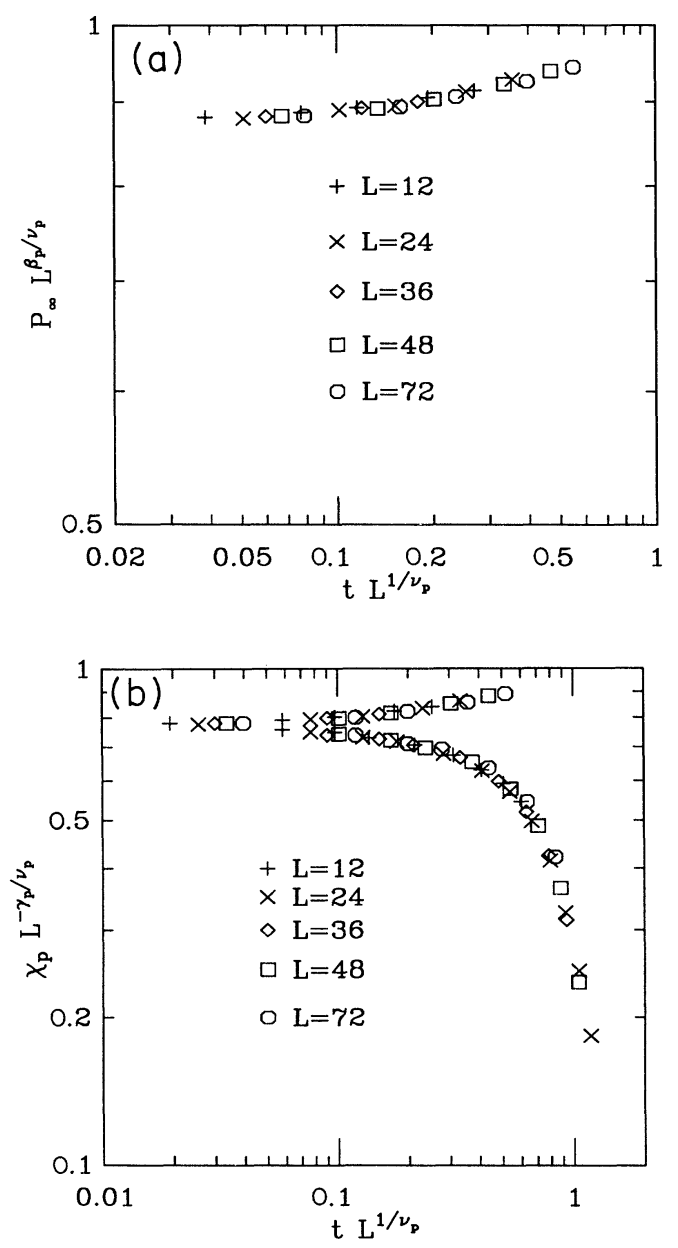

FIG. 3. Finite-size scaling plots for the FPT model, (a) $P_{\infty}$ and (b) $\chi_{p}$. and the hyperscaling law

$$
d v_{p}=\gamma_{p}+2 \beta_{p}
$$

The fractal dimension $D$ is obtained through the relation

$$
D=\left(\gamma_{p}+\beta_{p}\right) / \nu_{p} .
$$

We conclude that the Wolff clusters in an AFPT model are in the same universality class as random $2 \mathrm{D}$ percolation.

We next consider the FPT model with Hamiltonian (1) and $J=+1$. $T_{c}$ was previously determined to be $1.39 \pm 0.02 .^{18}$ Figure 3 shows the scaling plots for $P_{\infty}$ and $\chi_{p}$. (Attempts to make $n_{s}^{*}$ data collapse on a scaling plot were unsuccessful.) From these plots we determined $T_{p}$ to be $1.40 \pm 0.02$. Note that the uncertainty in $T_{p}$ in this case is much larger than that in the AFPT model. This is because $v_{p}$ is larger in the present case and consequently the coefficient $L^{1 / v_{p}}$ in the argument of the scaling functions (9) and (10) has a smaller range of accessible values, leading to a larger uncertainty in $t$.

The cluster exponents from the FPT model are also summarized in Table I. Hyperscaling (16) is again found to be satisfied numerically. Since we could only fit three independent exponents $\beta_{p}, v_{p}$, and $\gamma_{p}$ in this case, we cannot check the scaling laws (15). We observe that $T_{p}$ agrees with $T_{c}$ and the different exponent values show this system is in a different universality class from random 2D percolation. It is intriguing that $\beta_{p}, v_{p}$, and $\gamma_{p}$ are all larger than those of uncorrelated percolation by about the same factor $\sim 1.85$.

\section{DISCUSSION}

\section{A. AFPT mode}

In the AFPT model, we notice that $T_{p}>T_{c}$. The Wolff clusters percolate when the temperature is still appreciably higher than $T_{c}$. At temperatures near $T_{c}$, the cluster distribution is dominated by either very small clusters or infinite clusters. Thus, a great deal of computer time is spent in growing the infinite clusters, which, when flipped, have the net effect of updating those spins in the holes of the clusters only. This makes the algorithm very inefficient around $T_{c}$. At $T_{p}$, which is high compared with $T_{c}$, the spin correlation length is short and one may expect that the site-bond percolation resembles the random percolation. Hence, we expect the percolation of the Wolff clusters to be in the same universal class as the random 2D percolation, as confirmed in the exponents of the Wolff clusters.

\section{B. FPT model}

It is known from an exact mapping that, for the unfrustrated Ising and Potts model (where the acceleration algorithms has been successfully applied), $T_{p}=T_{c}{ }^{6,19}$ In the case of the ferromagnetic $X Y$ model, previous work ${ }^{4}$ showed numerically that the Wolff algorithm successfully accelerated the dynamics near $T_{c}$, and by the argument at the beginning of Sec. V (below), this indicates that 
$T_{p}=T_{c}$. Our result directly confirms that $T_{p}=T_{c}$ for the FPT model. At $T_{c}$ the spin-correlation length diverges and the site-bond percolation changes to another universality class, as shown in the exponents of the Wolff clusters.

In general dimension $d$, we can distinguish two scenarios for what limits the size of Wolff percolation clusters in the FPT model. Scenario 1: large clusters become exponentially unlikely as they involve the product of many probabilities which are small compared to unity. This case is familiar, since it occurs in ordinary uncorrelated percolation. Scenario 2: a FPT system can be broken into "domains" by "domain boundaries" with respect to some projection vector $r$. These are boundaries separating regions where the spin components along $\mathbf{r}$ are of opposite signs. Within a given domain, the projection of every spin onto the Wolff projection vector $\mathbf{r}$ has the same sign and the Wolff clusters cannot jump across the domain boundaries. ${ }^{20}$ Then, in this scenario, the clusters are limited by the domain boundaries, i.e., a typical Wolff cluster percolates and has a nonzero density through most of the interior of a domain.

Let us consider scenario 1. Since the cluster growth is not stopped by the domain boundaries but by the accumulation of the probabilities of broken bonds in the interior of the domains, we expect $\xi_{p}<\xi$. If $T_{p} \leq T_{c}$, we do not expect to find the same exponents as in ordinary percolation because the bond probabilities depend on the spin fluctuations, and near $T_{c}$ these develop long-range correlations. It has been shown ${ }^{21}$ that, for random bond percolation where the occupations $p_{x}$ have long-range two-point correlations

$$
\left\langle p_{x} p_{y}\right\rangle-p^{2} \sim|x-y|^{-a}
$$

the correlations are relevant (changing all the exponents) if $a<1 / v_{p}$. In this case, the new correlation length exponent is $v^{\prime}=1 / a>v_{p}$. The fact that we do not have an increased value of $v_{p}$ for the FPT case as compared to random percolation is consistent with this point of view, which would conclude that $a=1 / \nu_{p} \approx 0.8$. On the other hand, the KT theory ${ }^{12}$ implies a decay of spin correlations (due only to spin waves)

$$
\left\langle\sigma_{x} \sigma_{y}\right\rangle \sim|x-y|^{-\eta_{\mathrm{KT}}}
$$

with $\eta_{\mathrm{KT}}(T)=\frac{1}{4}$ at $T=T_{c}$. In view of the nonlinear relation (3) and the possible importance of higher than twopoint correlations, it is not clear to us how (18) might be related to (19).

Note that, in scenario 1, it would be somewhat surprising to find $T_{c}=T_{p}$ exactly. Even in the long-range correlated percolation models, the critical fraction $p_{c}$ is still between 0 and 1 . Thus, one would not expect the onset of long-range correlations in the percolation to make a sudden qualitative change so that the system reaches percolation. Therefore, the observation that $T_{p}=T_{c}$ argues strongly for scenario 2 .

Let us now consider scenario 2. For $d=2$, it has been noted by Brower et al. ${ }^{20}$ that every vortex must sit on a domain boundary; it is also clear that vortices of + and - signs must alternate along the domain boundary, and domain boundaries do not intersect. Then a single bound vortex pair typically produces a domain boundary loop so the domain diameter is comparable to the vortex spacing. Near $T_{c}$ where there are vortices on many length scales, we must be more careful about this picture. The loworder equations of the KT renormalization group implicitly assume a separation of length scales between vortices; given a cutoff $b$, it is assumed that all vortices with spacing closer than $b$ can be uniquely grouped into $(+,-)$ pairs and that they affect larger scales only by a renormalization of the effective spin stiffness constant. The corresponding domain picture is then hierarchically nested: every bound pair of two vortices must be connected by a domain boundary; smaller bound pairs can occur between the two vortices, but no larger bound pairs can occur between them. A schematic picture is shown in Fig. 4. It turns out that the KT correlation length is, in fact, the separation of the largest vortex pairs, i.e., the typical separation of unbound vortices. ${ }^{12}$ At larger scales the vortices are unbound and random. At this point the domain boundaries connect nearby vortices randomly and we expect the domain size to be comparable to the spacing of unbound vortices. But the percolation correlation length is the cutoff of the cluster distribution. Thus, within scenario 2 where clusters are identified with domains, we get $\xi_{p} \sim \xi$.

In the case of ordinary uncorrelated percolation (at criticality), if we rescale the system, the cluster distribution is constant. In other words, clusters have a self-similar distribution; the density of clusters of size greater than $R^{D}$ scales as $R^{-d}$, which implies

$$
n(s) \sim s^{-(d / D+1)},
$$

which, in turn, implies the hyperscaling relation. However, for vortex pairs in the KT theory, the vortex pair den-

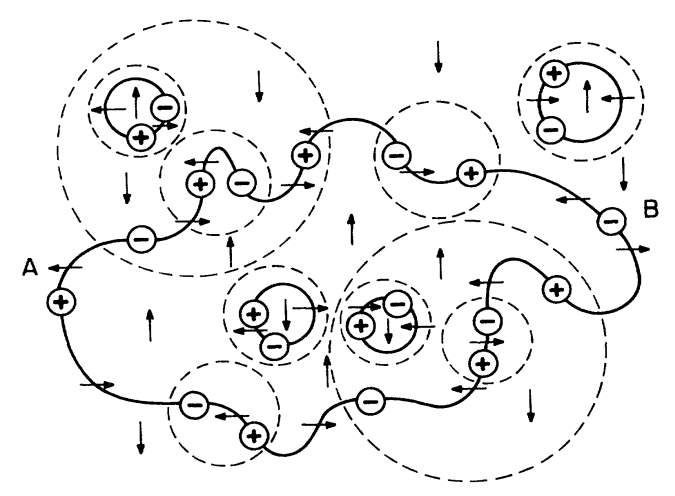

FIG. 4. Schematic picture of domain walls (solid lines) in a hierarchical configuration of bound vortices. Vortices are marked "+" and "-" according to their sign; a dashed circle encloses each bound pair. The Wolff projection vector $\mathbf{r}$ is taken to be vertical. The up and down arrows label domains in which the spins have positive and negative projections on $\mathbf{r}$; along the domain walls the spin directions are strictly horizontal, in the sense marked by the horizontal arrows. Under further coarse graining, this configuration would be mapped to a single loop of domain wall connecting vortices $A$ and $B$. 
sity is proportional to $y^{2}$, where $y=e^{-E_{c} / T}$ is the vortex fugacity and $E_{c}$ is the vortex core energy; the distribution can look the same on all length scales only if $y \rightarrow$ const. Now, the KT recursion relations ${ }^{12}$ have the form

$$
\begin{aligned}
& \frac{d y}{d l} \sim\left(T-T_{*}\right) y, \\
& \frac{d T}{d l} \sim y^{2},
\end{aligned}
$$

near the fixed point at $T_{*}$, where $b \sim e^{l}$ is the cutoff length scale. They iterate to a fixed point where $y=0$. So, under scenario 2 , it follows that the cluster distribution is not exactly self-similar but must be corrected by a prefactor $y^{2}$. To be more precise, at $T_{c}$, the point where Eq. (21) iterates to $T=T_{*}$, they turn out to have a singular solution $y(l) \sim 1 / l$. Thus, at $T_{c}$ the distribution of vortex pairs is self-similar except for a logarithmic prefactor $\sim 1 /(\ln R)^{2}$. Under scenario 2 , and assuming that the fractal dimension relation (14) is still valid, we note $\ln R \sim \ln s$ and so conclude that the cluster size distribution ought to have the form

$$
n(s) \sim s^{-(d / D+1)} /(\ln s)^{2} .
$$

Since (22) is just modified by a logarithmic correction, there is no important effect on other scaling assumptions and on exponent relations which did not involve $v_{p}$.

Under scenario 2, we must change the way $t$ enters in the scaling assumptions. The scaling assumptions (9) and (10) become

$$
\begin{aligned}
& P_{\infty}=L^{-x} f^{\prime}\left(e^{-c / t^{1 / 2}} L\right), \\
& \chi_{p}=L^{y} g^{\prime}\left(e^{-c / t^{1 / 2}} L\right),
\end{aligned}
$$

where $c$ is nonuniversal. The scaling plots of these forms are shown in Fig. 5. There is apparently no visible difference on the quality of the fits in Figs. 3 and 5. The exponents are found to be

$$
\begin{aligned}
& x=0.100 \pm 0.005, \\
& y=1.78 \pm 0.01, \\
& c=2.2 \pm 0.2 .
\end{aligned}
$$

The values of $\beta_{p} / v_{p}, \gamma_{p} / v_{p}$, and $v_{p}$ as determined from Fig. 3 are $0.100 \pm 0.005,1.80 \pm 0.01$, and $2.5 \pm 0.2$, respectively. Note that $x, y$, and $c$ are close in value to $\beta_{p} / v_{p}$, $\gamma_{p} / v_{p}$, and $v_{p}$ fit from the power-law form in Sec. III. In the KT theory,

$$
\chi \sim \xi^{2-\eta_{\mathrm{KT}}},
$$

where $2-\eta_{\mathrm{KT}}=1.75$. The closeness of $\gamma_{p} / \nu_{p}$ to 1.75 is intriguing. For the Ising model, it is known that $\chi_{p}=\chi$ (Ref. 19) and, in $d=2$, the Ising model also has $\gamma / v=1.75$. However, we are not seeing a simple coincidence with the Ising behavior since $v_{p} \approx 2.5 \neq v_{\text {Ising }}=1$. But there is no theory for why $\chi_{p} \sim \chi$ should be true in the $X Y$ case. It would be interesting to check whether $\gamma_{p} / \nu_{p}=\gamma / v$ in $d>2$ where there is an ordinary critical point.
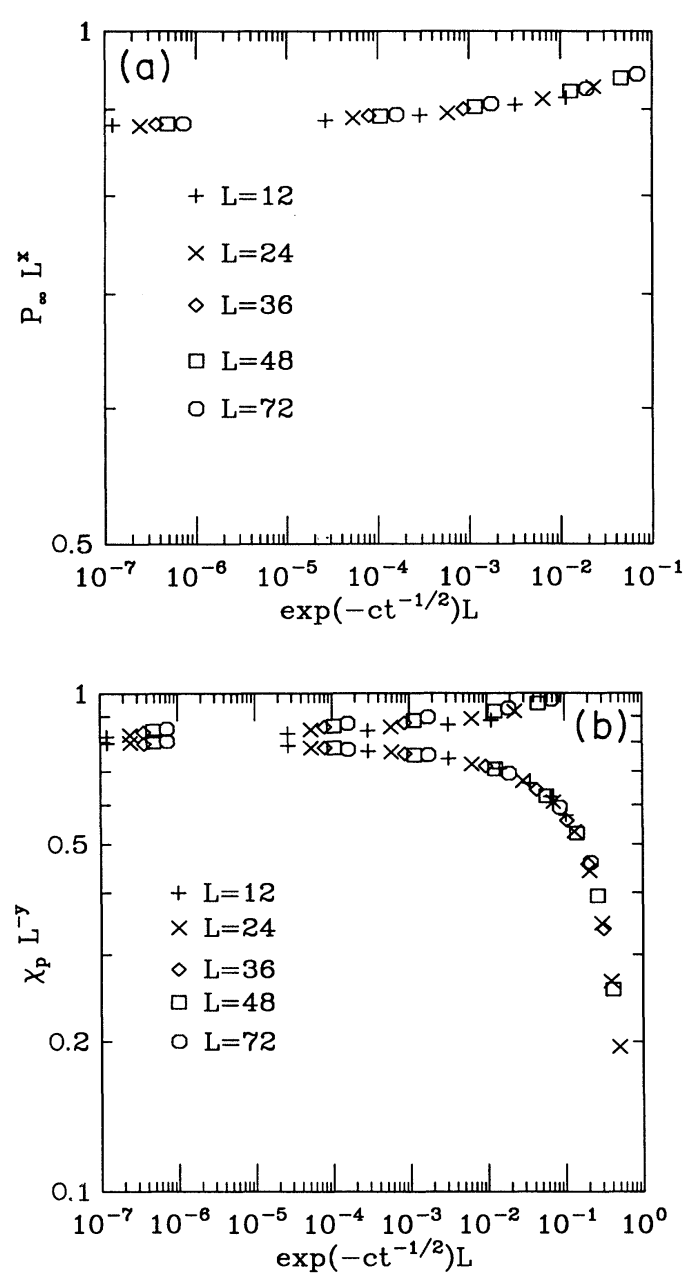

FIG. 5. Scaling plots for the FPT model using the KT form of the scaling relations.

\section{CONCLUSION}

By comparing our results on the AFPT and FPT models, a crucial condition for the success of the Wolff algorithm is how $T_{p}$ compares with $T_{c}$. If $T_{p}$ is higher than $T_{c}$, the Wolff clusters percolate before reaching $T_{c}$ as the temperature is decreased, as in the case of the AFPT model, and by the time we approach the point of most interest, $T_{c}$, the algorithm is very inefficient because the clusters are mostly spanning clusters. So we want $T_{p} \leq T_{c}$. But if $T_{p}$ is too low compared with $T_{c}$, the average cluster size at $T_{c}$ will be small and the algorithm will not be efficient. Based on this, one would guess that the algorithm is most efficient when $T_{c}=T_{p}$, in which case spanning clusters just start to appear at $T_{c}$.

In the FPT case, the geometrical insight gained from knowledge of the percolation properties improves our understanding of exactly what kind of degrees of freedom are being updated. Furthermore, a physical picture of what is stopping the growth of the clusters would be useful for possible modifications of the algorithm to deal 
with unfrustrated vector spins at $T<T_{c}$ (Ref. 22) or possibly with frustration.

We now consider the relation between the dynamic exponent $z_{W}$ and the exponents $\gamma_{p}$ and $\nu_{p}$. Tamayo et al. ${ }^{7}$ have presented essentially the same argument for the Ising case. The natural time unit for comparing computational work is one update per spin, which is proportional to the system size $N$. Since, in a Wolff update of a cluster of size $s$, only a fraction $s / N$ of the spins are updated, and $\langle s\rangle=\chi_{p}$, the Wolff correlation time $\tau_{W}$ has to be rescaled by a factor $\chi_{p} / N$ [Ref. 7, Eq. (9)]. Now, let us sit at $T_{p}$ and consider the time scale for updating the longest-wavelength mode. Clearly this should be related to the updates of spanning clusters. Each spanning cluster includes a fraction $\sim L^{-\beta_{p} / v_{p}}$ of all spins. We postulate the following assumption: when we hit each site (of order) once with a spanning cluster, the system completely forgets the old value of its longest-wavelength mode. This means we must hit a total of $L^{\beta_{p} / v_{p}}$ spanning clusters. Recall that the probability of hitting a cluster is proportional to its size; then, since the fraction of clusters we hit which are spanning is $L^{-\beta_{p} / v_{p}}$, we need a total of $L^{2 \beta_{p} / v_{p}}$ Wolff hits.

However, ${ }^{7}$ the next time a spin is hit by a spanning cluster, it is possible that this cluster comprises essentially the same sites as the previous time that the spin in question was hit; if so, this update merely undoes the effect of the earlier one. To correct for this, we must multiply the number of Wolff hits by $L^{z_{p}}$, where $z_{p}$ is the dynamic exponent for the percolation correlation function which measures the overlap between how the system decomposes into clusters at times zero and $t$ different times. This overlap is the analog of $\langle M(0) M(t)\rangle$ in a ferromagnetic spin system.

Finally, we conclude the time required to completely decorrelate the longest wavelength is

$$
\tau_{W}=L^{2 \beta_{p} / v_{p}+z_{p}} \frac{\chi_{p}}{N}
$$

i.e.,

$$
z_{W}=\left[\left(2 \beta_{p}+\gamma_{p}\right) / v_{p}-d\right]+z_{p} .
$$

The result of Ref. 7 for $z_{W}$ [their Eq. (15)] is the same as our Eq. (28) except it is in terms of the spin exponents, which are identical to the percolation exponents for the
Ising case. Our argument shows that, more generally, the percolation exponents, not the spin exponents, must be used.

If the percolation exponents satisfy hyperscaling, as we indeed found numerically (for $d=2$ ) in the FPT model, then (28) gives $z_{W}=z_{p}$. More generally, if hyperscaling is violated $^{23}$ we get

$$
z_{W}=\theta+z_{p}
$$

where $\theta \geq 0$ is the violation-of-hyperscaling exponent to be added to $d$ in (16) and (20).

For the Ising case, the Ising spin exponents and hence the Wolff percolation exponents satisfy hyperscaling, so $z_{W}=z_{p}$. Tamayo et al. ${ }^{7}$ measured the dynamic exponent $z_{\chi \chi}$ of the susceptibility correlation function $\langle\chi(0) \chi(t)\rangle$ which is apparently the percolation analog of $\left\langle M(0)^{2} M(t)^{2}\right\rangle$ in a spin system; this should have the same critical exponent $z_{\chi \chi} \equiv z_{p}$. Their numerical results show that $z_{p}=0$ for the $d=4$ Ising model, but $z_{p}>0$ for $d<4$.

For vector spins, it has already been observed by Brower et al. ${ }^{20}$ that $z_{W}=0$ for vector spins in the upper critical dimension $d=4$. Furthermore, their numerical results ${ }^{20}$ are consistent with $z_{W}=0$ in lower dimensions. Since both terms in (29) are non-negative for physical reasons, this would imply not only that the Wolff percolation satisfies hyperscaling, but that $z_{p}=0$ for all $d<4$.

To summarize, we have found $T_{p}$ and the cluster exponents for the FPT and AFPT models. In the case of the AFPT where the Wolff algorithm is unsuccessful in reducing critical slowing down, the Wolff clusters percolate at temperatures high above $T_{c}$, and the clusters are in the same universal class as random 2D percolations. In the case of the FPT where the Wolff algorithm is successful in reducing critical slowing down, we found that $T_{p}=T_{c}$ and the percolation of the Wolff clusters changed to another universal class.

\section{ACKNOWLEDGMENTS}

We would like to thank I. Clejan for sending us his Wolff algorithm code. C.L.H. is grateful to R. Brower for discussions and especially to I. Clejan and B. E. Larson for a related collaboration (Ref. 22) out of which this project developed. This research was supported by the Department of Energy Grant No. DE-FG-89ER45404.
${ }^{*}$ Present address: Supercomputer Computations Research Institute, Florida State University, Tallahassee, Florida 32306.

${ }^{1}$ R. Swendsen and J. S. Wang, Phys. Rev. Lett. 58, 86 (1987).

${ }^{2}$ D. Kandel, E. Domany, D. Ron, A. Brandt, and E. Loh, Jr., Phys. Rev. Lett. 60, 1591 (1988).

${ }^{3}$ F. Niedermayer, Phys. Rev. Lett. 61, 2026 (1988).

${ }^{4}$ U. Wolff, Phys. Rev. Lett. 62, 361 (1989).

5 J. S. Wang, R. H. Swendsen, and R. Kotecký, Phys. Rev. Lett. 63, 109 (1989).

${ }^{6}$ P. W. Kasteleyn and C. M. Fortuin, J. Phys. Soc. Jpn. Suppl. 26, 11 (1969); C. M. Fortuin and P. W. Kasteleyn, Physica (Utrecht) 57, 536 (1972).
${ }^{7}$ P. Tamayo, R. C. Brower, and W. Klein, J. Stat. Phys. 58, 1083 (1990).

${ }^{8}$ E. Dagotto and J. B. Kogut, Phys. Rev. Lett. 58, 299 (1987).

${ }^{9}$ G. G. Batrouni, E. R. Katz, A. S. Kronfeld, G. P. Lepage, B. Svetitsky, and K. G. Wilson, Phys. Rev. D 32, 2736 (1985).

${ }^{10}$ G. G. Batrouni and E. Dagotto, Phys. Rev. B 37, 9875 (1988); A. L. Scheinine, ibid. 39, 9368 (1989); B. E. Larson and C. L. Henley (unpublished).

${ }^{11}$ D. Stauffer, Phys. Rep. 54, 1 (1979).

12J. M. Kosterlitz and D. J. Thouless, J. Phys. C 6, 1181 (1973); D. R. Nelson, in Phase Transitions and Critical Phenomena, edited by C. Domb and J. L. Lebowitz (Academic, New York, 
1983), Vol. 7.

${ }^{13}$ D. H. Lee, J. D. Joannopoulos, J. W. Negele, and D. P. Landau, Phys. Rev. B 33, 450 (1986). See also, S. Miyashita and H. Shiba, J. Phys. Soc. Jpn. 53, 1145 (1984).

${ }^{14}$ M. P. M. den Nijs, J. Phys. A 12, 1857 (1979).

${ }^{15}$ R. B. Pearson, Phys. Rev. B 22, 2579 (1980).

${ }^{16}$ B. Nienhuis, E. K. Riedel, and M. Schick, J. Phys. A 13, L189 (1980).

${ }^{17} \mathrm{M}$. Sahimi, in The Mathematics and Physics of Disordered Media, edited by A. Dold and B. Eckmann (Springer-Verlag, New York, 1983).
${ }^{18}$ F. Falo, L. M. Floria, and R. Navarro, J. Phys. Condens. Matter 1, 5139 (1989).

${ }^{19}$ A. Coniglio and W. Klein, J. Phys. A 13, 2775 (1980).

${ }^{20}$ R. C. Brower, N. A. Gross, and K. J. M. Moriarty (unpublished).

${ }^{21}$ A. Weinrib, Phys. Rev. B 29, 387 (1984).

${ }^{22}$ I. Clejan, C. L. Henley, and B. E. Larson (unpublished).

${ }^{23}$ This occurs in the "self-organized percolation" model which has a dynamics violating detailed balance [C. L. Henley (unpublished)]. 